Barker, P. F., Kennett, J. P., et al., 1990

Proceedings of the Ocean Drilling Program, Scientific Results, Vol. 113

\title{
54. SILICEOUS SPONGE SPICULES FROM OCEAN DRILLING PROGRAM LEG 1131
}

\author{
Kevin McCartney²
}

\begin{abstract}
Siliceous sponge spicules are present throughout many of the sections drilled by Ocean Drilling Program Leg 113. The assemblages consist mostly of monaxons and occur in Eocene to Pleistocene strata. Occurrences of the various spicule types are tabulated for Sites $689,693,694,695,696$, and 697.
\end{abstract}

\section{INTRODUCTION}

Siliceous sponge spicules occur throughout much of ODP Sites 689, 693, 694, 695, 696, and 697 of Leg 113 off the East coast of Antarctica and the Antarctic Peninsula (Fig. 1), and were tabulated while the sediment samples were being examined for silicoflagellates (McCartney and Wise, this volume). The assemblages are dominated by monaxons, with other types representing only a minor fraction of the total abundance.

There has been relatively little study of sponge spicules done on sediment samples obtained from deep sea drilling. The DSDP work on sponge spicules are summarized in Table 1 (some of these papers contain little except a few photographs). This lack of spicule study is not due to scarcity, as sponge spicules are often quite abundant, but is because of inability to make detailed taxonomic distinctions based upon isolated spicules. This makes the biostratigraphic utilization and environmental interpretation of isolated sponge spicules a seemingly impossible task, and thus few paleontologists choose to spend time on them. But while specific spicule morphologies do not now have much use in geologic interpretation, their general occurrence may be of some significance. Furthermore, the variety of sponge spicules found in deep-sea sediment samples may be of interest to micropaleontologists.

\section{METHODS}

For Sites 689 and 690, in which there was an abundance of calcareous material, raw samples were placed in $100 \mathrm{~mL}$ beakers and a small amount of $30 \%$ hydrogen peroxide added. After 2$4 \mathrm{hr}$, the beaker was placed in an ultrasonic cleaner and distilled water was added to about the $25 \mathrm{~mL}$ level. $\mathrm{HCl}$ was added and the beaker heated for $30 \mathrm{~min}$. Samples were then centrifuged and decanted twice and washed once before making strewn slides. For Sites 693 to 697 , in which there was little calcareous material, strewn slides were made directly.

Sponge spicules were counted while examining the slide for silicoflagellates. In general, an entire $22 \times 40 \mathrm{~mm}$ slide was examined for each sample (for exceptions, see McCartney and Wise, this volume). Sponge spicules were usually not abundant enough for relative counts, as done by McCartney (1987). Instead, the overall abundance of sponge spicules (Tables 2-7) is indicated by the following scale: Abundant $(A)=100-1000$ spicule specimens; Common $(C)=10-100$ specimens; Uncommon $(U)=1-10$ specimens; Not found $(N)=0$ specimens. Approximate counts include fragments.

\footnotetext{
${ }^{1}$ Barker, P. F., Kennett, J. P., et al., 1990. Proc. ODP, Sci. Results, 113: College Station, TX (Ocean Drilling Program).

2 Department of Geology, Florida State University, Tallahassee, Florida 32306. Current address: Division of Mathematics and Science, University of Maine at Presque Isle, Presque Isle, ME 04769.
}

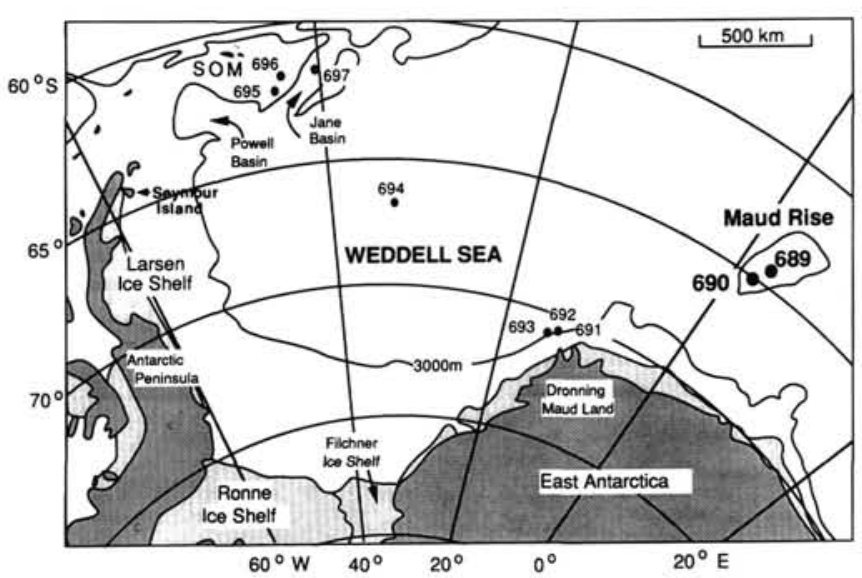

Figure 1. Location map showing positions of Sites $689,693,694,695$, 696, and 697 in the Weddell Sea.

\section{SITE SUMMARIES}

\section{Hole 689B (Table 2)}

Hole $689\left(64^{\circ} 31.009^{\prime} \mathrm{S}, 03^{\circ} 05.996^{\prime} \mathrm{E}\right.$; water depth, $\left.2080 \mathrm{~m}\right)$ was drilled near the crest of Maud Rise. It is isolated from any influence of terrigenous sedimentation from the East Antarctic continent besides windblown fine clastics and ice-rafted debris. This site produced the most complete section of siliceous microfossil-bearing sediment samples, with ages extending to the Eocene. Sponge spicules, especially oxeas, are present in the Pliocene to Eocene strata and are particularly abundant and diverse in the upper Oligocene. Spicular frameworks are relatively abundant in the lower Oligocene and Eocene samples.

\section{Hole 690B}

Hole 690B $\left(65^{\circ} 09.629^{\prime} \mathrm{S}, 01^{\circ} 12.296^{\prime} \mathrm{E}\right.$; water depth $\left.2914 \mathrm{~m}\right)$ was drilled on the southwestern flank of the Maud Rise, in the eastern Weddell Sea. It was drilled, with Site 689, as part of a two-hole transect to study the history of the vertical water mass stratification on Maud Rise. Sponge spicules are very sparse, and consist of only an occasional oxea. Because of the rarity of sponge spicules, a table showing occurrence is not included in this paper (see McCartney and Wise, this volume, for a list of samples studied).

\section{Hole 693A (Table 3)}

Hole $693 \mathrm{~A}\left(70^{\circ} 49.892^{\prime} \mathrm{S}, 14^{\circ} 34.410^{\prime} \mathrm{W}\right.$; water depth, $\left.2359 \mathrm{~m}\right)$ was drilled into a mid-slope bench on the Weddell Sea margin of East Antarctica (see Fig. 1). Sponge spicules were found to be sparse in the Pleistocene and Pliocene but more abundant in the 
Table 1. DSDP reports in which siliceous sponge spicules are discussed or illustrated, with Leg number and location.

\begin{tabular}{cll}
\hline Leg & \multicolumn{1}{c}{ Location } & \multicolumn{1}{c}{ Author } \\
\hline 13 & Northeast Atlantic & Dumitrica (1973) \\
44 & Northwest Atlantic & Bukry (1978) \\
49 & Mid-Atlantic & Bukry (1979) \\
$51-53$ & Northwest Atlantic & Bukry (1980a) \\
54 & East Equatorial Pacific & Bukry (1980b) \\
71 & South Atlantic & Ivanik (1983) \\
90 & Southwest Pacific & Locker and Martini (1986) \\
93 & Northwest Atlantic & McCartney (1987) \\
\hline
\end{tabular}

Miocene and Oligocene samples. As with Site 689, the spicules are especially abundant and diverse in the upper Oligocene. Non-monaxon spicules are uncommon except in Sample 113693A-40R-1, 30-32 cm.

\section{Holes 694B and 649C (Table 4)}

Holes 649B ( $66^{\circ} 50.835^{\prime} \mathrm{S}, 33^{\circ} 26.826^{\prime} \mathrm{W}$; water depth, $\left.4653 \mathrm{~m}\right)$ and $694 \mathrm{C}\left(66^{\circ} 50.820^{\prime} \mathrm{S}, 33^{\circ} 26.763^{\prime} \mathrm{W}\right.$; water depth, $\left.4653 \mathrm{~m}\right)$ were drilled on the northern part of the Weddell Sea abyssal plain, and are remote from terrestrial areas. While silicoflagellates are especially rare at this site (see McCartney and Wise, this volume), siliceous sponge spicules are relatively common. The spicules are almost exclusively monaxon.

\section{Hole 695A (Table 5)}

Hole $695 \mathrm{~A}\left(62^{\circ} 23.476^{\prime} \mathrm{S}, 43^{\circ} 27.095^{\prime} \mathrm{W}\right.$; water depth, $\left.1305 \mathrm{~m}\right)$ was drilled on the southern margin of the South Orkney microcontinent, in the northern Weddell Sea. Sponge spicules were found to be relatively abundant and especially diverse throughout the Pleistocene and Pliocene (this is very different from what was found in the earlier holes of Leg 113). Isochelae were more abundant at this site than any other except Site 696.

\section{Holes 696A and 696B (Table 6)}

Holes $696 \mathrm{~A}\left(61^{\circ} 50.945^{\prime} \mathrm{S}, 42^{\circ} 55.984\right.$; water depth, $\left.650 \mathrm{~m}\right)$ and $696 \mathrm{~B}\left(61^{\circ} 50.959^{\prime} \mathrm{S}, 42^{\circ} 55.996^{\prime} \mathrm{W}\right.$; water depth, $\left.650 \mathrm{~m}\right)$ were drilled on the southeast margin of the South Orkney microcontinent. The Pliocene interval has sponge spicules of abundance and diversity very similar to that found in Hole 695A. The upper Miocene samples are similar to the Pliocene but contain an unusual abundance of dichotrianes.

\section{Hole 697B (Table 7)}

Hole $697 \mathrm{~B}\left(61^{\circ} 48.626^{\prime} \mathrm{S}, 40^{\circ} 17.749^{\prime} \mathrm{W}\right.$; water depth 3483$)$ is the northernmost hole of a three-hole transect that includes Sites 695 and 696. It also lies in the deepest water of the three. Sponge spicules are generally less abundant than at Sites 695 or 696 and also less diverse, with fewer non-monaxons. Isochelae are much less abundant than at Sites 695 and 696.

Table 2. Relative abundance of Eocene to Pleistocene sponge spicules from ODP Hole 689B, Cores 113-689B-1H to -15H. The occurrence of "actino-" specimens are designated by " $\mathrm{a}$ ".

\begin{tabular}{|c|c|c|c|c|c|c|c|c|c|c|c|c|}
\hline Age & $\begin{array}{l}\text { Core, section, } \\
\text { interval }(\mathrm{cm})\end{array}$ & 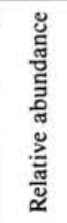 & हैँ & $\frac{\mathscr{e}}{\sum_{n}}$ & 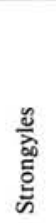 & 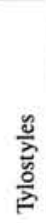 & 高 & 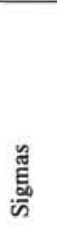 & 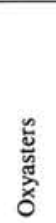 & 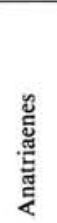 & 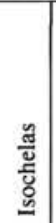 & 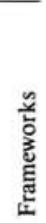 \\
\hline Pliocene & $\begin{array}{l}1 \mathrm{H}-1,29-31 \\
1 \mathrm{H}-4,29-31 \\
2 \mathrm{H}-2,33-55 \\
2 \mathrm{H}-5,29-31 \\
3 \mathrm{H}-2,30-32\end{array}$ & $\begin{array}{l}\mathrm{N} \\
\mathrm{N} \\
\mathrm{N} \\
\mathrm{N} \\
\mathrm{U}\end{array}$ & $\mathrm{x}$ & & & & & & & & & \\
\hline Miocene & $\begin{array}{l}3 \mathrm{H}-5,29-31 \\
4 \mathrm{H}-2,29-31 \\
4 \mathrm{H}-5,30-32 \\
5 \mathrm{H}-2,30-32 \\
5 \mathrm{H}-5,29-31 \\
6 \mathrm{H}-2,30-32 \\
6 \mathrm{H}-5,29-31 \\
7 \mathrm{H}-2,29-31 \\
7 \mathrm{H}-5,29-31 \\
8 \mathrm{H}-2,30-32\end{array}$ & $\begin{array}{c}\mathrm{N} \\
\mathrm{U} \\
\mathrm{U} \\
\mathrm{C} \\
\mathrm{N} \\
\mathrm{C} \\
\mathrm{U} \\
\mathrm{U} \\
\mathrm{C} / \mathrm{U} \\
\mathrm{N}\end{array}$ & $\begin{array}{l}\mathrm{X} \\
\mathrm{X} \\
\mathrm{X} \\
\mathrm{X} \\
\mathrm{X} \\
\mathrm{X} \\
\mathrm{X}\end{array}$ & $\mathrm{x}$ & & & & $\begin{array}{l}X \\
X \\
X\end{array}$ & $\mathrm{x}$ & & & $\mathrm{x}$ \\
\hline $\begin{array}{c}\text { late } \\
\text { Oligocene }\end{array}$ & $\begin{array}{r}8 \mathrm{H}-5,30-32 \\
9 \mathrm{H}-2,29-31 \\
9 \mathrm{H}-5,29-31 \\
10 \mathrm{H}-2,29-31 \\
10 \mathrm{H}-5,29-31 \\
11 \mathrm{H}-2,32-34 \\
11 \mathrm{H}-5,32-34 \\
12 \mathrm{H}-2,29-31 \\
\end{array}$ & $\begin{array}{l}\text { A } \\
\text { C } \\
\text { A } \\
\text { A } \\
\text { C } \\
\text { A } \\
\text { C } \\
\text { C }\end{array}$ & $\begin{array}{l}\mathrm{X} \\
\mathrm{X} \\
\mathrm{X} \\
\mathrm{X} \\
\mathrm{X} \\
\mathrm{X} \\
\mathrm{X} \\
\mathrm{X} \\
\mathrm{X}\end{array}$ & $\begin{array}{l}\mathrm{Xa} \\
\mathrm{X} \\
\mathrm{X} \\
\mathrm{X}\end{array}$ & $\begin{array}{l}X \\
X\end{array}$ & $\begin{array}{l}x \\
x \\
x\end{array}$ & $\mathrm{x}$ & $\mathrm{x}$ & $x$ & $\mathrm{X}$ & $\begin{array}{l}\mathrm{x} \\
\mathrm{x}\end{array}$ & $\begin{array}{l}\mathrm{X} \\
\mathrm{X} \\
\mathrm{X} \\
\mathrm{X} \\
\mathrm{X} \\
\end{array}$ \\
\hline $\begin{array}{c}\text { early } \\
\text { Oligocene }\end{array}$ & $\begin{array}{l}12 \mathrm{H}-5,29-31 \\
13 \mathrm{H}-2,29-31 \\
13 \mathrm{H}-5,28-30 \\
14 \mathrm{H}-2,30-32\end{array}$ & $\begin{array}{l}\mathrm{C} \\
\mathrm{C} \\
\mathrm{C} \\
\mathrm{C}\end{array}$ & $\begin{array}{l}\mathrm{x} \\
\mathrm{x} \\
\mathrm{x} \\
\mathrm{x}\end{array}$ & & & & & $\mathrm{x}$ & & & & $\begin{array}{l}\mathrm{x} \\
\mathrm{x} \\
\mathrm{x} \\
\end{array}$ \\
\hline Eocene & $\begin{array}{l}14 \mathrm{H}-5,30-32 \\
15 \mathrm{H}-2,30-32\end{array}$ & $\begin{array}{l}\mathrm{C} \\
\mathrm{C}\end{array}$ & $\begin{array}{l}\mathrm{X} \\
\mathrm{X}\end{array}$ & & & & & & & & $\mathrm{x}$ & $\begin{array}{l}\mathrm{X} \\
\mathrm{X}\end{array}$ \\
\hline
\end{tabular}


Table 3. Relative abundance of Oligocene to Pleistocene sponge spicules from ODP Hole 693A, Cores 113-693A-3R to -40R. The occurrence of "actino-" specimens are designated by "a".

\begin{tabular}{|c|c|c|c|c|c|c|c|c|}
\hline Age & $\begin{array}{l}\text { Core, section, } \\
\text { interval }(\mathrm{cm})\end{array}$ & 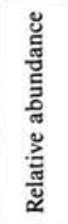 & ॐّँ & $\frac{\mathscr{c}}{\grave{n}}$ & 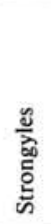 & 䒕 & 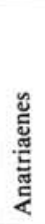 & 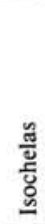 \\
\hline Pleistocene & $3 R-2,124-126$ & $\mathrm{~N}$ & & & & & & \\
\hline $\begin{array}{c}\text { late } \\
\text { Pliocene }\end{array}$ & $\begin{array}{l}4 \mathrm{R}-2,30-32 \\
5 \mathrm{R}-2,30-32 \\
6 \mathrm{H}-2,30-32\end{array}$ & $\begin{array}{l}\mathrm{N} \\
\mathrm{N} \\
\mathrm{N}\end{array}$ & & & & & & \\
\hline $\begin{array}{c}\text { early } \\
\text { Pliocene }\end{array}$ & $\begin{array}{l}8 \mathrm{R}-2,30-32 \\
9 \mathrm{R}-2,30-32 \\
10 \mathrm{R}-2,30-32 \\
11 \mathrm{R}-3,30-32 \\
12 \mathrm{R}-2,28-30 \\
13 \mathrm{R}-2,30-32 \\
14 \mathrm{R}-2,30-32 \\
15 \mathrm{R}-2,30-32 \\
17 \mathrm{R}-3,30-32 \\
18 \mathrm{R}-2,30-32 \\
19 \mathrm{R}-2,30-32\end{array}$ & $\begin{array}{l}\mathrm{N} \\
\mathrm{N} \\
\mathrm{U} \\
\mathrm{U} \\
\mathrm{N} \\
\mathrm{N} \\
\mathrm{U} \\
\mathrm{U} \\
\mathrm{U} \\
\mathrm{U} \\
\mathrm{U}\end{array}$ & $\begin{array}{l}\mathrm{X} \\
\mathrm{X} \\
\\
\mathrm{Xa} \\
\mathrm{X} \\
\mathrm{X} \\
\mathrm{X} \\
\mathrm{X}\end{array}$ & & & & & $x$ \\
\hline $\begin{array}{c}\text { late } \\
\text { Miocene }\end{array}$ & $\begin{array}{l}21 \mathrm{R}-2,28-30 \\
22 \mathrm{R}-2,30-32 \\
25 \mathrm{R}-2,30-32 \\
26 \mathrm{R}-1,30-32 \\
27 \mathrm{R}-2,30-32 \\
28 \mathrm{R}-2,30-32 \\
29 \mathrm{R}-2,30-32\end{array}$ & $\begin{array}{l}\mathrm{C} / \mathrm{U} \\
\mathrm{C} \\
\mathrm{U} \\
\mathrm{C} \\
\mathrm{U} \\
\mathrm{C} \\
\mathrm{C}\end{array}$ & $\begin{array}{l}\mathrm{X} \\
\mathrm{X} \\
\mathrm{X} \\
\mathrm{X} \\
\mathrm{X} \\
\mathrm{X} \\
\mathrm{x}\end{array}$ & & & $\mathrm{x}$ & & \\
\hline $\begin{array}{c}\text { early } \\
\text { Miocene }\end{array}$ & $\begin{array}{l}31 \mathrm{R}-3,30-32 \\
33 \mathrm{R}-1,96-98\end{array}$ & $\begin{array}{l}\mathrm{C} \\
\mathrm{C}\end{array}$ & $\begin{array}{l}\mathrm{x} \\
\mathrm{x}\end{array}$ & & $\mathrm{x}$ & & & $\mathrm{x}$ \\
\hline $\begin{array}{c}\text { late } \\
\text { Oligocene }\end{array}$ & $\begin{array}{l}34 \mathrm{R}-2,118-120 \\
35 \mathrm{R}-2,30-32 \\
36 \mathrm{R}-1,30-32 \\
37 \mathrm{R}-1,34-36\end{array}$ & $\begin{array}{l}\mathrm{C} \\
\mathrm{C} \\
\mathrm{C} \\
\mathrm{C}\end{array}$ & $\begin{array}{l}\mathrm{X} \\
\mathrm{X} \\
\mathrm{X} \\
\mathrm{X}\end{array}$ & $\begin{array}{l}\mathrm{x} \\
\mathrm{x}\end{array}$ & $x$ & $\mathrm{x}$ & & \\
\hline $\begin{array}{l}\text { middle-late } \\
\text { Oligocene }\end{array}$ & $\begin{array}{l}38 \mathrm{R}-1,92-94 \\
39 \mathrm{R}-1,30-32 \\
40 \mathrm{R}-1,30-32\end{array}$ & $\begin{array}{l}\mathrm{C} \\
\mathrm{C} \\
\mathrm{A}\end{array}$ & $\begin{array}{l}\mathrm{X} \\
\mathrm{X} \\
\mathrm{Xa}\end{array}$ & $\begin{array}{l}\mathrm{X} \\
\mathrm{X} \\
\mathrm{Xa}\end{array}$ & $\mathrm{x}$ & $\mathrm{X}$ & $\mathrm{X}$ & $\mathrm{X}$ \\
\hline
\end{tabular}

\section{DESCRIPTION OF SPONGE SPICULES}

Although a Linnaean taxonomy for isolated sponge spicules was used by early workers, a descriptive terminology is now preferred (see Hyman, 1940; Bukry, 1978; Ivanik, 1983). Modern poriferan taxonomy depends upon the disposition of spicules in the skeletal framework; a typical sponge can possess a wide variety of spicule types (see, for example, Hartman, 1958). In addition, very different sponges can have individual spicules that are nearly identical (Levi, 1957). For these reasons, a formal taxonomy based on isolated spicules is impractical. There is a natural tendency, however, for the micropaleontologist to try to at least incorporate higher levels of taxonomy into the systematics (see McCartney, 1987), but even this is difficult in the context of sponge spicules. While some spicule morphologies, such as amphidiscs or hexactines, are relatively restricted taxonomically, the usage in this paper will be entirely descriptive.

\section{Monaxons}

\section{Diactinal (monaxons with similar ends)}

Oxeas (pointed ends; Pl. 1, Fig. 1): Very abundant, generally making up the majority of the sponge spicules in each sample; it is often the only spicule type found. The length and relative width are quite variable. Specimens can be longer than $450 \mu \mathrm{m}$. Relatively few specimens had a noticeable axial canal, caused by
Table 4. Relative abundance of Pliocene and Pleistocene sponge spicules from ODP Hole 694B, Cores 113-694B-5X to $-24 X$, and $113-694 \mathrm{C}-2 \mathrm{X}$ to $-14 \mathrm{X}$. The occurrence of "actino-" specimens are designated by "a".

\begin{tabular}{|c|c|c|c|c|c|c|c|}
\hline Age & $\begin{array}{l}\text { Core, section, } \\
\text { interval }(\mathrm{cm})\end{array}$ & 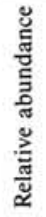 & 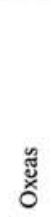 & 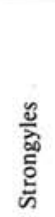 & $\frac{\tilde{e}}{\sqrt{2}}$ & 莺 & 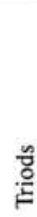 \\
\hline $\begin{array}{c}\text { early } \\
\text { Pliocene }\end{array}$ & $\begin{array}{r}\text { 694B-5X-1, 80-82 } \\
6 \mathrm{X}-2,22-24 \\
7 \mathrm{H}-4,28-30 \\
9 \mathrm{H}-1,8-10 \\
13 \mathrm{H}-1,28-30 \\
14 \mathrm{H}-1,75-77\end{array}$ & $\begin{array}{l}\mathrm{N} \\
\mathrm{N} \\
\mathrm{N} \\
\mathrm{N} \\
\mathrm{N} \\
\mathrm{U}\end{array}$ & $\mathrm{X}$ & $=$ & & $\mathrm{X}$ & \\
\hline $\begin{array}{c}\text { late } \\
\text { Miocene }\end{array}$ & $\begin{array}{l}19 \mathrm{H}-1,14-16 \\
22 \mathrm{X}-1,39-41 \\
23 \mathrm{X}-1,29-31 \\
24 \mathrm{X}-3,132-134\end{array}$ & $\begin{array}{l}\mathrm{N} \\
\mathrm{C} \\
\mathrm{U} \\
\mathrm{N}\end{array}$ & $\begin{array}{l}\mathrm{X} \\
\mathrm{X}\end{array}$ & & & & \\
\hline $\begin{array}{l}\text { middle } \\
\text { Miocene }\end{array}$ & $\begin{array}{r}694 C-2 X-1,26-28 \\
5 X-2,28-30 \\
6 X-2,30-32 \\
7 X-2,59-61 \\
8 X-1,30-32 \\
9 X-1,53-55 \\
10 X-1,28-30 \\
11 X-2,30-32 \\
12 X-1,29-32 \\
13 X-1,30-32 \\
14 X-2,88-89\end{array}$ & $\begin{array}{l}\mathrm{N} \\
\mathrm{U} \\
\mathrm{C} \\
\mathrm{U} \\
\mathrm{C} \\
\mathrm{C} \\
\mathrm{C} \\
\mathrm{C} \\
\mathrm{A} \\
\mathrm{C} \\
\mathrm{A}\end{array}$ & $\begin{array}{l}\mathrm{X} \\
\mathrm{X} \\
\mathrm{X} \\
\mathrm{X} \\
\mathrm{X} \\
\mathrm{Xa} \\
\mathrm{X} \\
\mathrm{X} \\
\mathrm{X} \\
\mathrm{X}\end{array}$ & $\begin{array}{l}\mathrm{X} \\
\mathrm{X} \\
\mathrm{X} \\
\mathrm{X}\end{array}$ & $\mathrm{x}$ & $\mathrm{X}$ & $\begin{array}{l}X \\
X \\
X\end{array}$ \\
\hline
\end{tabular}

dissolution (see de Laubenfels, 1955). Acanthoxeas were rare (marked by "a" in occurrence listings).

Strongyles (monaxons with rounded ends; Pl. 1, Fig. 2): When found, strongyles were sparse. Variability of size and surface texture were generally similar to co-occurring oxeas and styles. Acanthostrongyles are relatively more abundant than acanthoxeas.

\section{Monactinal (monaxons rounded at one end and pointed at the other)}

Styles (no change in thickness at rounded end; Pl. 1, Figs. 3, 4): Styles were consistently less abundant than oxeas, with seldom more than 10 styles in a single slide. Variation in size and relative width similar to that found with oxeas. Specimens often have an obvious hollow area with the spicule (see illustrated specimens).

Tylostyles (one end pointed, the other knobbed; Pl. 2, Fig. 6): Occurred sparsely. Acanthotylostyles (Pl. 2, Fig. 4) are marked by " $a$ " in occurrence listings.

\section{Polyaxons (many equal-sized rays radiating from a single point)}

Spherasters and oxyasters (ball-like spicules with pointed spines; Pl. 2, Fig. 1; Pl. 3, Fig. 4): Both spherasters and oxyasters were rare. There is a wide variation in size, as shown by the illustrated specimens.

Amphiasters (having spines that radiate from the ends of a short axis): a single specimen of this type was found in Sample 113-697B-17X-1, 31-33 cm.

\section{Tetraxons and Triaxons}

Triods (three rays of equal size; Pl. 2, Fig. 2): The illustrated specimen is of exceptional size.

Dichotriaenes (triradial symmetry with forked rays; PI. 2, Figs. 5, 7, Pl. 3, Figs. 2, 3): There is wide variation in size, as 
Table 5. Relative abundance of Pliocene and Pleistocene sponge spicules from ODP Hole 695A, Cores 113-695A-1H to -36X. The occurrence of "actino-" specimens are designated by " $\mathrm{a}$ ".

\begin{tabular}{|c|c|c|c|c|c|c|c|c|c|}
\hline Age & $\begin{array}{l}\text { Core, section, } \\
\text { interval }(\mathrm{cm})\end{array}$ & 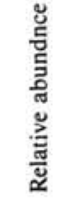 & ญै & $\frac{\tilde{d}}{\bar{n}}$ & $\begin{array}{l}\frac{\mathscr{d}}{2} \\
\frac{0}{2}\end{array}$ & 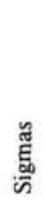 & 造 & 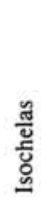 & 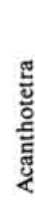 \\
\hline Pleistocene & $\begin{array}{l}1 \mathrm{H}-2,30-32 \\
2 \mathrm{H}-2,30-32\end{array}$ & $\begin{array}{l}\mathrm{C} \\
\mathrm{C}\end{array}$ & $\begin{array}{l}\mathrm{X} \\
\mathrm{X}\end{array}$ & $\mathrm{X}$ & & $\begin{array}{l}\mathrm{X} \\
\mathrm{X}\end{array}$ & & $\mathrm{x}$ & \\
\hline $\begin{array}{c}\text { late } \\
\text { Pliocene }\end{array}$ & $\begin{array}{l}3 \mathrm{H}-2,30-32 \\
4 \mathrm{H}-2,30-32 \\
5 \mathrm{H}-2,30-32 \\
6 \mathrm{H}-2,30-32\end{array}$ & $\begin{array}{c}\mathrm{C} / \mathrm{U} \\
\mathrm{C} \\
\mathrm{U} \\
\mathrm{C}\end{array}$ & $\begin{array}{l}\mathrm{X} \\
\mathrm{X} \\
\mathrm{X}\end{array}$ & $\begin{array}{l}\mathrm{X} \\
\mathrm{x}\end{array}$ & $\mathrm{X}$ & & & & \\
\hline $\begin{array}{c}\text { early } \\
\text { Pliocene }\end{array}$ & $\begin{array}{l}7 \mathrm{H}-2,31-33 \\
8 \mathrm{H}-2,130-132 \\
9 \mathrm{H}-2,30-32 \\
10 \mathrm{H}-2,30-32 \\
12 \mathrm{H}-2,30-32 \\
13 \mathrm{H}-2,30-32 \\
14 \mathrm{H}-2,28-30 \\
15 \mathrm{H}-2,30-32 \\
18 \mathrm{X}-2,30-32 \\
19 \mathrm{X}-2,30-32 \\
20 \mathrm{X}-2,31-33 \\
21 \mathrm{X}-2,30-32 \\
22 \mathrm{X}-2,30-32 \\
23 \mathrm{X}-2,30-32 \\
25 \mathrm{X}-2,30-32 \\
26 \mathrm{X}-2,30-32 \\
27 \mathrm{X}-2,130-132 \\
28 \mathrm{X}-2,34-36 \\
29 \mathrm{X}-2,30-32 \\
30 \mathrm{X}-2,30-32 \\
33 \mathrm{X}-2,30-32 \\
34 \mathrm{X}-2,58-60 \\
35 \mathrm{X}-2,34-36 \\
36 \mathrm{X}-2,27-30\end{array}$ & $\begin{array}{l}\mathrm{U} \\
\mathrm{U} \\
\mathrm{U} \\
\mathrm{U} \\
\mathrm{C} \\
\mathrm{U} \\
\mathrm{C} \\
\mathrm{C} \\
\mathrm{C} \\
\mathrm{C} \\
\mathrm{C} \\
\mathrm{C} \\
\mathrm{C} \\
\mathrm{C} \\
\mathrm{C} \\
\mathrm{U} \\
\mathrm{C} \\
\mathrm{C} \\
\mathrm{U} \\
\mathrm{C} \\
\mathrm{C} \\
\mathrm{C} \\
\mathrm{C} \\
\mathrm{C}\end{array}$ & $\begin{array}{l}\mathrm{X} \\
\mathrm{X} \\
\mathrm{X} \\
\mathrm{X} \\
\mathrm{X} \\
\mathrm{X} \\
\mathrm{X} \\
\mathrm{X} \\
\mathrm{X} \\
\mathrm{X} \\
\mathrm{X} \\
\mathrm{X} \\
\mathrm{X} \\
\mathrm{X} \\
\mathrm{X} \\
\mathrm{X} \\
\mathrm{X} \\
\mathrm{X} \\
\mathrm{X} \\
\mathrm{X} \\
\mathrm{X} \\
\mathrm{X} \\
\mathrm{X}\end{array}$ & $\begin{array}{l}\mathrm{X} \\
\mathrm{X} \\
\mathrm{X}\end{array}$ & X & $\begin{array}{l}\mathrm{X} \\
\mathrm{X} \\
\mathrm{X} \\
\mathrm{X}\end{array}$ & $\mathrm{X}$ & $\begin{array}{l}\mathrm{X} \\
\mathrm{X} \\
\mathrm{X} \\
\mathrm{X}\end{array}$ & $\mathrm{X}$ \\
\hline
\end{tabular}

shown by the illustrated specimens. Dichotriaenes were especially abundant in Hole 696B, where they were relatively small (see Pl. 3, Figs. 2-3).

Anatriaenes (having a shape similar to a three-armed anchor; Pl. 1, Figs. 5, 7): The occurrence of this spicule type was rare.

Amphidises (rod with recurved, sometimes petaled, disc on each end; Pl. 2, Fig. 3).

\section{Miscellaneous other spicule types}

Sigmas (strongly curved, or "C"-shaped spicules; Pl. 3, Figs. 1, 5): A wide variety of $\mathrm{C}$-shaped spicules were found. Their occurrence was always sparse, with never more than a few occurring on a single slide. Both ends of the spicule were usually curved within the same plane.

Isochelae (curved bar with terminal elaborations): Isochelae occurred sparsely in many samples. Some specimens were of relatively large size $(>40 \mu \mathrm{m})$. Specimens with anchorate (Pl. 3, Figs. 9-12) and unguiferate (Pl. 3, Figs. 7, 8; see also Hartman, 1982) terminations were both relatively common. In some cases, one termination would be somewhat smaller than the other.
Discorhabd (Pl. 3, Fig. 6): A single specimen of this type was found in Site 696B (see Hartman, 1982, fig. 16-19 for a similar specimen).

Frameworks (connected rods that form a net-like lattice; Pl. 1, Fig. 6).

\section{ACKNOWLEDGMENTS}

I would like to thank Dr. Sherwood W. Wise, Jr. for his encouragement and Sigurd Locker, Suzanne O'Connell, and James Kennett for constructive comments. Dennis Cassidy and the resources of the Antarctic Research Facility and Reference Library were of considerable help. Technical assistance was supplied by Kristin Magnison and Gerald Arnold. Laboratory support was provided by NSF Grant DPP- 8414268 and USSAC funds. In addition to the Leg 113 material I would like to thank the Deep Sea Drilling Project for providing samples from other legs for comparative study.

\section{REFERENCES}

Bukry, D., 1978. Cenozoic coccolith and silicoflagellate stratigraphy, Deep Sea Drilling Project Leg 44. In Benson, W. E., Sheridan, R. E., et al., Init. Repts. DSDP, 44: Washington (U.S. Govt. Printing Office), 807-864.

1979. Coccolith and silicoflagellate stratigraphy, northern Mid-Atlantic Ridge and Reykjanes Ridge, Deep Sea Drilling Project Leg 49. In Luyendyk, B. P., Cann, J. R., et al., Init. Repts. DSDP, 49: Washington (U.S. Govt. Printing Office), 551-582.

1980a. Eocene diatoms and siliceous sponge spicules from the northwestern Atlantic Ocean, DSDP Sites 417 and 418. In Donnelly, T., Francheteau, J., et al., Init. Repts. DSDP, 51, 52, 53: Washington (U.S. Govt. Printing Office), 851-855.

$1980 \mathrm{~b}$. Silicoflagellate biostratigraphy and paleoecology in the Eastern equatorial Pacific, Deep Sea Drilling Project Leg 54. In Rosendahl, B. R., Hekinian, R., et al., Init. Repts. DSDP, 54: Washington (U.S. Govt. Printing Office), 545-573.

Dumitrica, P., 1973. Cretaceous and Quaternary radiolaria in deep sea sediments from the northwest Atlantic Ocean and Mediterranean Sea. In Ryan, W. B. F., Hsü, K. J., et al., Init. Repts. DSDP, 13: Washington (U.S. Govt. Printing Office), 829-901.

Hartman, W. D., 1958. Natural history of the marine sponges of southern New England. Bull. Peabody Mus. Nat. Hist., 12:1-155. 1982. Form and distribution of silica in sponges. In Simpson, T. L., and Volcani, B. E. (Eds.), Silicon and Siliceous Structures in Biological Systems: New York (Springer-Verlag), 453-491.

Hyman L. H., 1940. The Invertebrates: Protozoa through Ctenophora: New York (McGraw-Hill).

Ivanik, M. M., 1983. Paleogene and Neogene sponge spicules from Sites 511, 512, and 513 in the South Atlantic. In Ludwig, W. J., Krasheninnikov, V. A., et al., Init. Repts. DSDP, 71: Washington (U.S. Govt. Printing Office), 933-950.

Laubenfels, M. W. de, 1955. Porifera. In Moore, R. C., Treatise on Invertebrate Paleontology, Lawrence (Univ. of Kansas Press), E21-E112.

Levi, C., 1957. Ontogeny and systematics in sponges. Syst. Zool. 6: 174-183.

Locker, S., and Martini, E., 1986. Silicoflagellates and some sponge spicules from the southwest Pacific, Deep Sea Drilling Project, Leg 90. In Kennett, J. P., von der Borch, C. C., et al., Init. Repts. DSDP, 90:. Washington (U.S. Govt. Printing Office), 887-924.

McCartney, K., 1987. Siliceous sponge spicules from Deep Sea Drilling Project Leg 93. In van Hinte, J. E., Wise, S. W., Jr., et al., Init. Repts. DSDP, 93: Washington (U.S. Govt. Printing Office), 815-824.

Date of initial receipt: 11 July 1988

Date of acceptance: 14 September 1989

Ms 113B-144 
Table 6. Relative abundance of Miocene and Pliocene sponge spicules from ODP Hole 696A (Cores 113-696A-1H to -9H) and 696B (Cores 113-696A-2R to -34R). The occurrence of "actino-" specimens are designated by "a".

\begin{tabular}{|c|c|c|c|c|c|c|c|c|c|c|c|}
\hline Age & $\begin{array}{l}\text { Core, section, } \\
\text { interval }(\mathrm{cm})\end{array}$ & 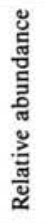 & ฮัँ & $\frac{u}{\grave{n}}$ & 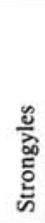 & $\begin{array}{l}\frac{\tilde{c}}{2} \\
\frac{\tilde{c}}{2} \\
\frac{0}{2}\end{array}$ & 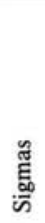 & 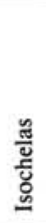 & 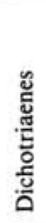 & 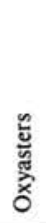 & 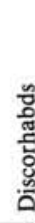 \\
\hline $\begin{array}{c}\text { late } \\
\text { Pliocene }\end{array}$ & $\begin{array}{r}696 \mathrm{~A}-1 \mathrm{H}-2,30-32 \\
2 \mathrm{H}-2,30-32 \\
3 \mathrm{H}-1,37-39 \\
4 \mathrm{H}-1,90-92 \\
5 \mathrm{H}-2,30-32 \\
6 \mathrm{H}-2,30-32 \\
7 \mathrm{H}-2,30-32 \\
8 \mathrm{H}-2,30-32 \\
9 \mathrm{H}-2,30-32 \\
696 \mathrm{~B}-2 \mathrm{R}-1,74-76\end{array}$ & $\begin{array}{l}\mathrm{C} \\
\mathrm{C} \\
\mathrm{C} \\
\mathrm{C} \\
\mathrm{C} \\
\mathrm{U} \\
\mathrm{U} \\
\mathrm{U} \\
\mathrm{A} \\
\mathrm{U}\end{array}$ & $\begin{array}{l}\mathrm{Xa} \\
\mathrm{Xa} \\
\mathrm{X} \\
\mathrm{X} \\
\mathrm{X} \\
\mathrm{X} \\
\mathrm{X} \\
\mathrm{X} \\
\mathrm{X} \\
\mathrm{X}\end{array}$ & $\begin{array}{l}x \\
x\end{array}$ & $\mathrm{X}$ & $\mathrm{Xa}$ & $\begin{array}{l}x \\
x \\
x\end{array}$ & $\begin{array}{l}\mathrm{X} \\
\mathrm{X} \\
\mathrm{X} \\
\mathrm{X}\end{array}$ & & & \\
\hline $\begin{array}{l}\text { early } \\
\text { Pliocene }\end{array}$ & $\begin{array}{l}3 \mathrm{R}-1,129-131 \\
5 \mathrm{R}-2,30-32 \\
6 \mathrm{R}-2,30-32 \\
7 \mathrm{R}-2,34-36 \\
10 \mathrm{R}-1,31-33 \\
13 \mathrm{R}-1,42-44 \\
17 \mathrm{R}-1,130-132 \\
19 \mathrm{R}-1,16-18 \\
20 \mathrm{R}-1,130-132\end{array}$ & $\begin{array}{l}\mathrm{C} \\
\mathrm{N} \\
\mathrm{C} \\
\mathrm{C} \\
\mathrm{C} \\
\mathrm{C} \\
\mathrm{C} \\
\mathrm{C} \\
\mathrm{C}\end{array}$ & $\begin{array}{l}\mathrm{x} \\
\mathrm{x} \\
\mathrm{x} \\
\mathrm{x} \\
\mathrm{X} \\
\mathrm{X} \\
\mathrm{x}\end{array}$ & $\mathrm{Xa}$ & $x$ & & $\begin{array}{l}\mathrm{X} \\
\mathrm{X} \\
\mathrm{X}\end{array}$ & $\begin{array}{l}x \\
x \\
x\end{array}$ & $\mathrm{X}$ & $x$ & \\
\hline $\begin{array}{c}\text { late } \\
\text { Miocene }\end{array}$ & $\begin{array}{l}24 R-2,30-32 \\
26 R-2,30-32 \\
27 R-2,30-32 \\
28 R-2,30-32 \\
32 R-2,30-32 \\
34 R-2,30-32\end{array}$ & $\begin{array}{l}\mathrm{C} \\
\mathrm{C} \\
\mathrm{C} \\
\mathrm{C} \\
\mathrm{C} \\
\mathrm{C}\end{array}$ & $\begin{array}{l}\mathrm{X} \\
\mathrm{X} \\
\mathrm{X} \\
\mathrm{X} \\
\mathrm{X} \\
\mathrm{X}\end{array}$ & $x$ & $X$ & $\begin{array}{c}\mathrm{X} \\
\mathrm{Xa}\end{array}$ & $\begin{array}{l}X \\
x\end{array}$ & $\mathrm{x}$ & $\begin{array}{l}\mathrm{X} \\
\mathrm{X} \\
\mathrm{X} \\
\mathrm{X} \\
\mathrm{X}\end{array}$ & & $\mathrm{x}$ \\
\hline
\end{tabular}

Table 7. Relative abundance of Pliocene and Pleistocene sponge spicules from ODP Hole 697B, Cores 113-697B-1H to -32X. The occurrence of "actino-" specimens are designated by "a".

\begin{tabular}{|c|c|c|c|c|c|c|c|c|c|}
\hline Age & $\begin{array}{l}\text { Core, section, } \\
\text { interval (cm) }\end{array}$ & 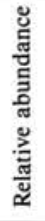 & đ̊ँ & $\frac{2}{2}$ & 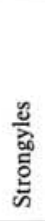 & 离 & 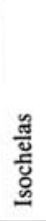 & $\begin{array}{l}\text { 妾 } \\
\text { 冚 } \\
\text { 范 }\end{array}$ & 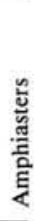 \\
\hline Pleistocene & $\begin{array}{r}697 \mathrm{~B}-1 \mathrm{H}-2,72-74 \\
2 \mathrm{H}-2,30-32 \\
3 \mathrm{H}-3,30-32 \\
4 \mathrm{H}-3,30-32 \\
\end{array}$ & $\begin{array}{l}\mathrm{N} \\
\mathrm{U} \\
\mathrm{U} \\
\mathrm{N}\end{array}$ & $\begin{array}{l}X \\
X\end{array}$ & & & & & & \\
\hline $\begin{array}{c}\text { early } \\
\text { Pliocene }\end{array}$ & $\begin{array}{l}6 \mathrm{H}-2,30-32 \\
7 \mathrm{H}-3,30-32 \\
8 \mathrm{H}-3,30-32 \\
9 \mathrm{H}-2,140-141 \\
11 \mathrm{H}-3,30-32 \\
13 \mathrm{X}-1,30-32 \\
14 \mathrm{X}-4,30-32 \\
16 \mathrm{X}-4,28-30 \\
17 \mathrm{X}-1,31-33 \\
19 \mathrm{X}-1,27-29 \\
20 \mathrm{X}-3,28-30 \\
21 \mathrm{X}-2,28-30 \\
22 \mathrm{X}-2,28-30 \\
25 \mathrm{X}-1,117-119 \\
26 \mathrm{X}-2,31-33 \\
27 \mathrm{X}-1,28-30 \\
28 \mathrm{X}-5,30-32 \\
29 \mathrm{X}-1,29-31 \\
30 \mathrm{X}-3,31-33 \\
31 \mathrm{X}-2,28-30 \\
32 \mathrm{X}-3,28-30 \\
32 \mathrm{X}-5,28-30\end{array}$ & $\begin{array}{l}\mathrm{U} \\
\mathrm{C} \\
\mathrm{U} \\
\mathrm{C} \\
\mathrm{C} \\
\mathrm{U} \\
\mathrm{U} \\
\mathrm{U} \\
\mathrm{C} \\
\mathrm{C} \\
\mathrm{C} \\
\mathrm{C} \\
\mathrm{C} \\
\mathrm{C} \\
\mathrm{N} \\
\mathrm{C} \\
\mathrm{C} \\
\mathrm{U} \\
\mathrm{U} \\
\mathrm{U} \\
\mathrm{U} \\
\mathrm{U}\end{array}$ & $\begin{array}{l}\mathrm{X} \\
\mathrm{X} \\
\mathrm{X} \\
\mathrm{Xa} \\
\mathrm{X} \\
\mathrm{X} \\
\mathrm{X} \\
\mathrm{Xa} \\
\mathrm{X} \\
\mathrm{X} \\
\mathrm{X} \\
\mathrm{X} \\
\mathrm{X} \\
\mathrm{X} \\
\mathrm{X} \\
\mathrm{X} \\
\mathrm{X} \\
\mathrm{X} \\
\mathrm{X} \\
\mathrm{X}\end{array}$ & $\begin{array}{c}\text { Xa } \\
\text { Xa } \\
\text { X } \\
\text { X } \\
\\
\text { X } \\
\text { X } \\
\text { X } \\
\text { X } \\
\\
\text { X }\end{array}$ & $\mathrm{X}$ & $\begin{array}{l}\mathrm{X} \\
\mathrm{X} \\
\mathrm{X}\end{array}$ & 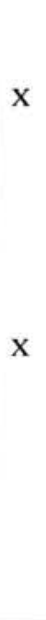 & $\begin{array}{l}\mathrm{X} \\
\mathrm{X} \\
\mathrm{X}\end{array}$ & $\mathrm{X}$ \\
\hline
\end{tabular}



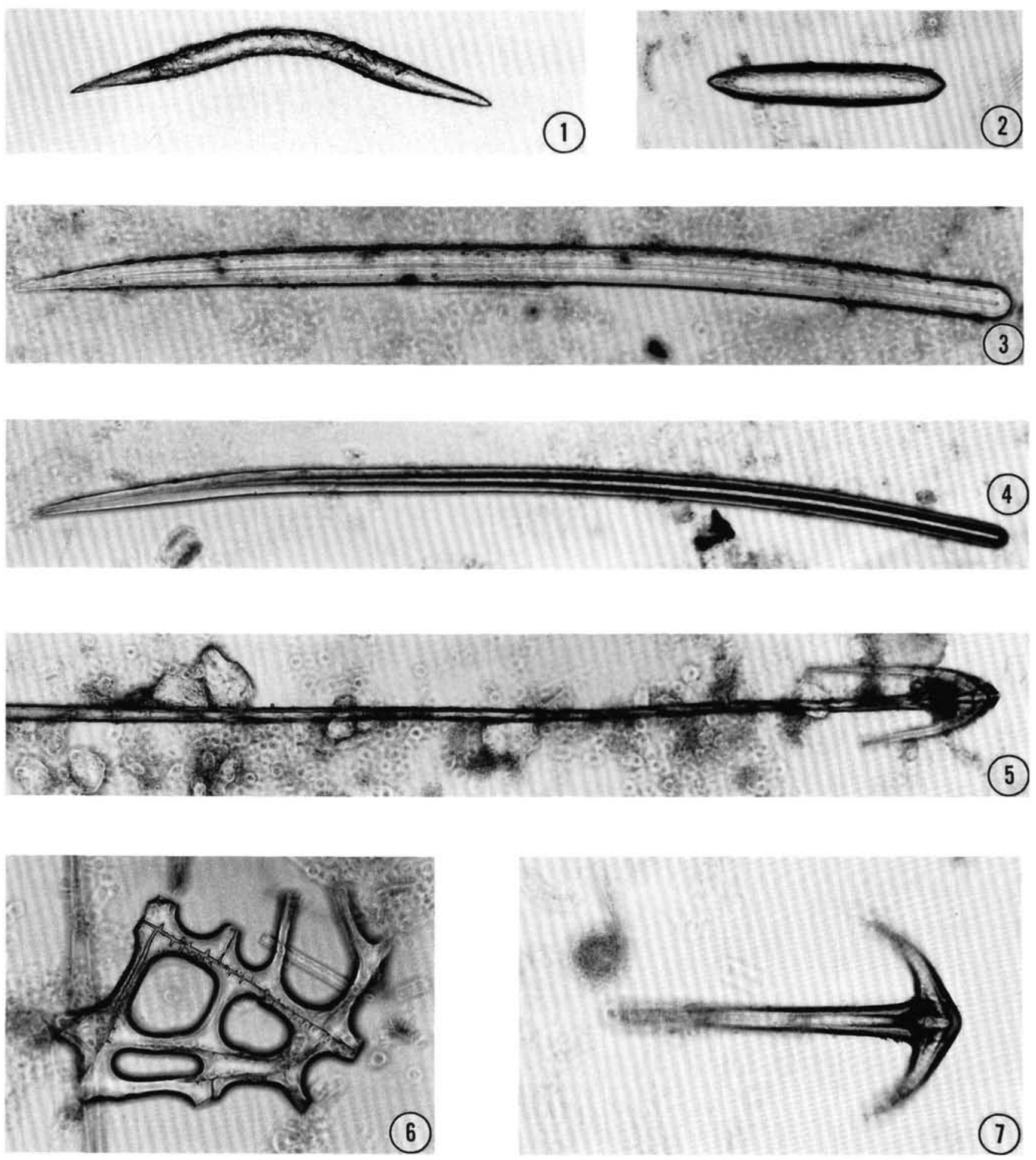

Plate 1. Siliceous sponge spicules from ODP Leg 113 (Magnification $330 \times$ ) 1. Oxea, Sample 113-689B-9H-5, 29-31 cm. 2. Strongyle, Sample 113-689B-9H-5, 29-31 cm. 3-4. Styles, (3) Sample 113-697B-17X-1, 31-33 cm; (4) Sample 113-697B-19X-1, 27-29 cm. 5. Anatriaene (?), Sample 113-695A-12H-2, 30-32 cm. 6. Framework, Sample 113-689B-8H-5, 30-32 cm. 7. Anatriaene, Sample 113-689B-8H-5, 30-32 cm. 

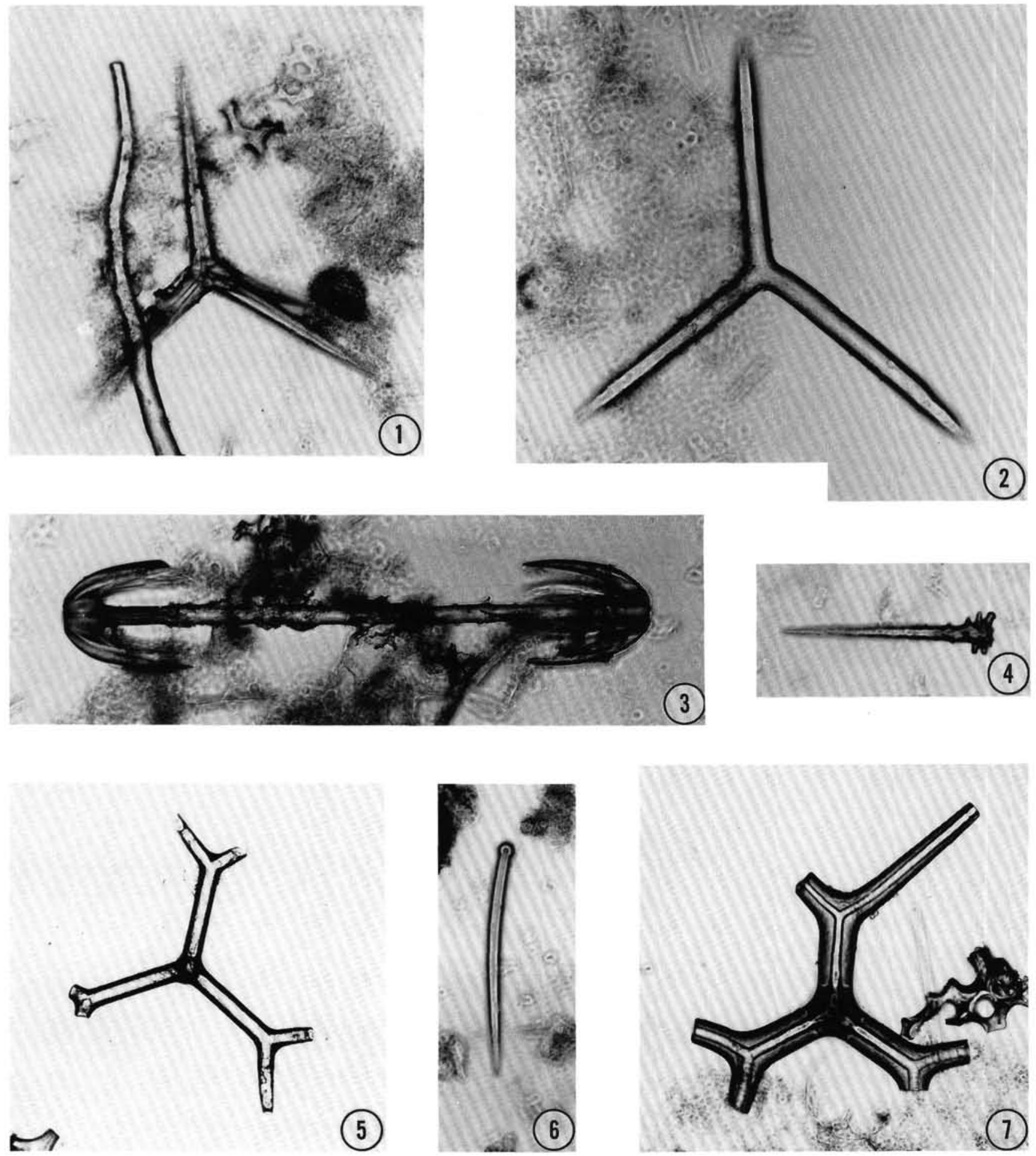

Plate 2. Siliceous sponge spicules from ODP Leg 113 (Magnification $330 \times$ ) 1. Oxyaster, Sample 113-689B-9H-5, 29-31 cm. 2. Triod, Sample 113-689B-10H-2, 32-34 cm. 3. Amphidisc, Sample 113-689B-14H-5, 30-32 cm. 4. Acanthotylostyle, Sample 113-696A-1H-2, 30-32 cm. 5. Dichotriaene, Sample 113-689B-12H-5, 29-31 cm. 6. Tylostyle, Sample 113-695A-22X-2, 30-32 cm. 7. Dichotriaene, Sample 113-689B-10H-2, $29-31 \mathrm{~cm}$. 

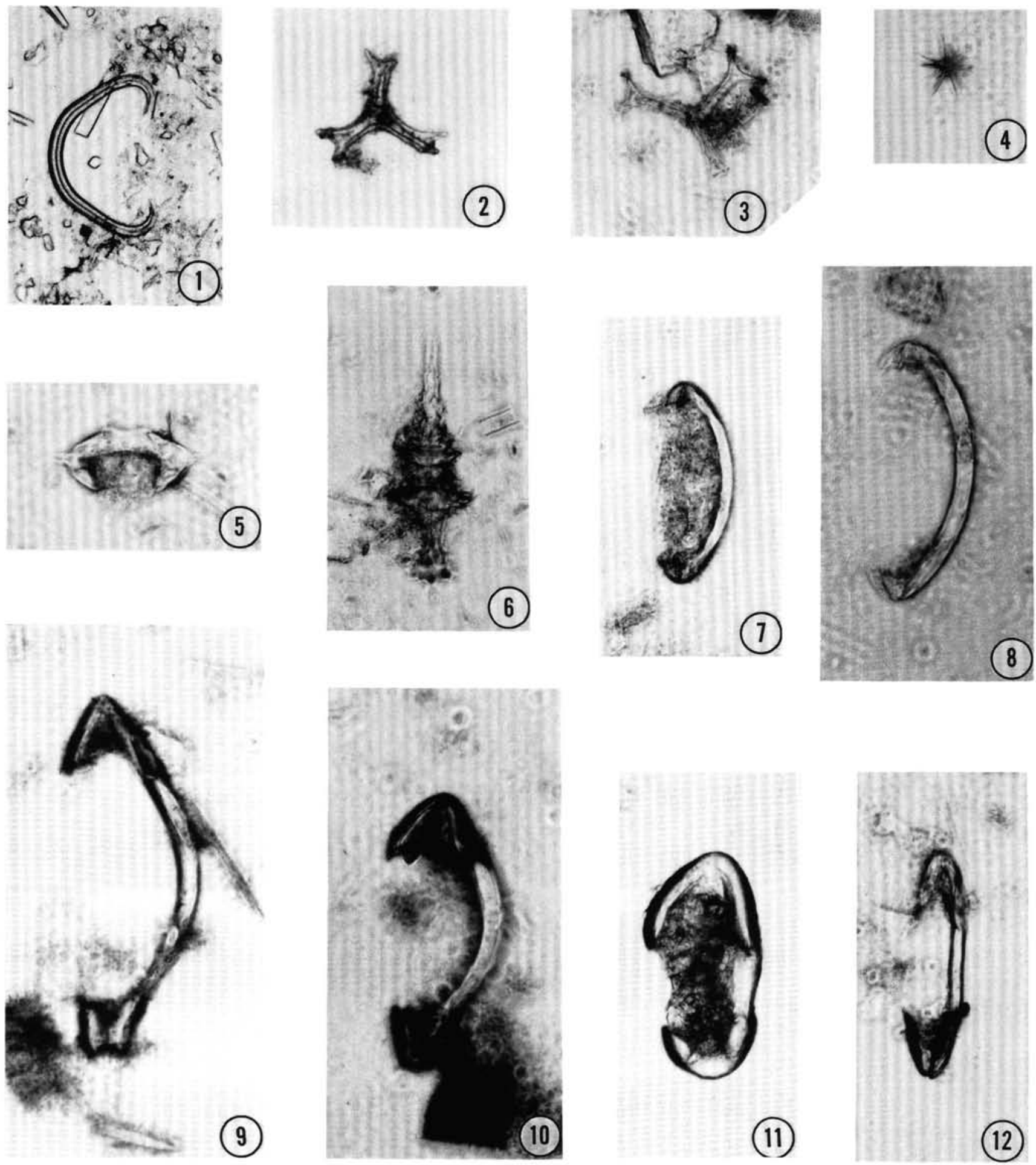

Plate 3. Siliceous sponge spicules from ODP Leg 113 (Magnification $490 \times$ ) 1. Sigma, Sample 113-689B-10H-2, 32-34 cm. 2-3. Dichotriaenes, (2) Sample 113-696B-24R-2, 30-32 cm; (3) Sample 113-696B-27R-2, 30-32 cm. 4. Oxyaster, Sample 113-696B-6R-2, 30-32 cm. 5. Sigma, Sample 113-696B-3R-2, 129-131 cm. 6. Discorhabd, Sample 113-696B-3R-2, 129-131 cm. 7-12. Isochelas, (7) Sample 113-696A-35-2, 34-36 cm; (8) Sample 113-697B-22X-2, 28-30 cm; (9) Sample 113-696B-20R-1, 130-132 cm; (10) Sample 113-696A-4H-1, 90-92 cm; (11) Sample 113-696B-7R-2, $34-36 \mathrm{~cm}$; (12) Sample 113-696A-9H-2, 30-32 cm. 